\title{
Large Cell Neuroendocrine Carcinoma Harboring an Anaplastic Lymphoma Kinase (ALK) Rearrangement with Response to Alectinib
}

\author{
Nobuyoshi Hayashi ${ }^{1,2}$, Akihisa Fujita ${ }^{1}$, Toyohiro Saikai ${ }^{1}$, Hirotugu Takabatake ${ }^{1}$, \\ Mie Sotoshiro $^{1}$, Kyuutarou Sekine ${ }^{1}$ and Akihiko Kawana ${ }^{2}$
}

\begin{abstract}
:
Anaplastic lymphoma kinase (ALK) rearrangement is most commonly observed in lung adenocarcinoma in a subset of lung cancer. Large cell neuroendocrine carcinoma (LCNEC) harboring an ALK rearrangement is very rare. Based on the findings from a transbronchial lung biopsy, a 75-year-old non-smoking woman was diagnosed with LCNEC with multiple liver and bone metastases. After seven cycles of cytotoxic chemotherapy, her genotype testing demonstrated ALK rearrangement. Subsequently, she was administered alectinib and exhibited a partial response.
\end{abstract}

Key words: LCNEC, pulmonary neuroendocrine carcinoma, ALK rearrangement and alectinib

(Intern Med 57: 713-716, 2018)

(DOI: 10.2169/internalmedicine.9368-17)

\section{Introduction}

Although lung cancer is a major cause of death from cancer in Japan, anaplastic lymphoma kinase (ALK) inhibitors have produced remarkable clinical responses compared with those produced by standard cytotoxic chemotherapy in nonsmall cell lung cancer (NSCLC) harboring an ALK rearrangement $(1,2)$. Large cell neuroendocrine carcinoma (LCNEC) is a relatively rare lung tumor. Pulmonary LCNEC is biologically aggressive, similarly to small cell lung cancer (SCLC). The survival curves of pulmonary LCNEC and SCLC overlap at every stage, and the survival is lower in them than in other NSCLCs (3). To our knowledge, only two cases of a pulmonary LCNEC harboring an ALK rearrangement have been reported $(4,5)$.

We herein report a case of pulmonary LCNEC harboring an ALK rearrangement that responded to alectinib.

\section{Case Report}

A 75-year-old non-smoking woman was diagnosed with liver hemangioma in 2011 and subsequently underwent an abdominal ultrasonography (US) scan every year. An abdominal US scan revealed multiple nodules in the liver, and she was referred to our hospital in January 2016. Chest computed tomography (CT) revealed a 21-mm large, lobulated nodule in the left lower lobe (Fig. 1A), and abdominal CT revealed multiple liver metastases (Fig. 1B). Laboratory data demonstrated elevated tumor maker levels [pro-gastrinreleasing peptide (ProGRP), $942.2 \mathrm{pg} / \mathrm{mL}$ (normal range, 0$46 \mathrm{pg} / \mathrm{mL}$ ) and neuron-specific enolase (NSE), $30.1 \mathrm{ng} / \mathrm{mL}$ (normal range, $0-1 \mathrm{ng} / \mathrm{mL}$ )]. A maximum-intensity projection image from ${ }^{18} \mathrm{~F}$-fluorodeoxiglucose positron emission tomography $\left({ }^{18} \mathrm{~F}-\mathrm{FDG}\right.$ PET) demonstrated an intense radiotracer uptake in the left lower lobe, left hilar lymph node, medial lymph node, both sides of the rib, and liver (Fig. 2A). A transbronchial lung biopsy specimen from the lower lobe revealed malignant neoplasia organized into solid nests of tumor cells with moderately abundant cytoplasm (Fig. 3A). The tumor cells tested positive for CD56 (Fig. 3B), chromogranin (Fig. 3C), and synaptophysin (Fig. 3D) and negative for thyroid transcription factor-1 (TTF-1) in immunohistochemical studies (Fig. 3E). Thus,

${ }^{1}$ Department of Respiratory Medicine, Sapporo Minamisanjyou Hospital, Japan and ${ }^{2}$ Division of Infectious Disease and Pulmonary Medicine, Department of Internal Medicine, National Defense Medical College, Japan

Received: April 19, 2017; Accepted: June 26, 2017; Advance Publication by J-STAGE: November 20, 2017

Correspondence to Dr. Nobuyoshi Hayashi, n-hayashi@ndmc.ac.jp 

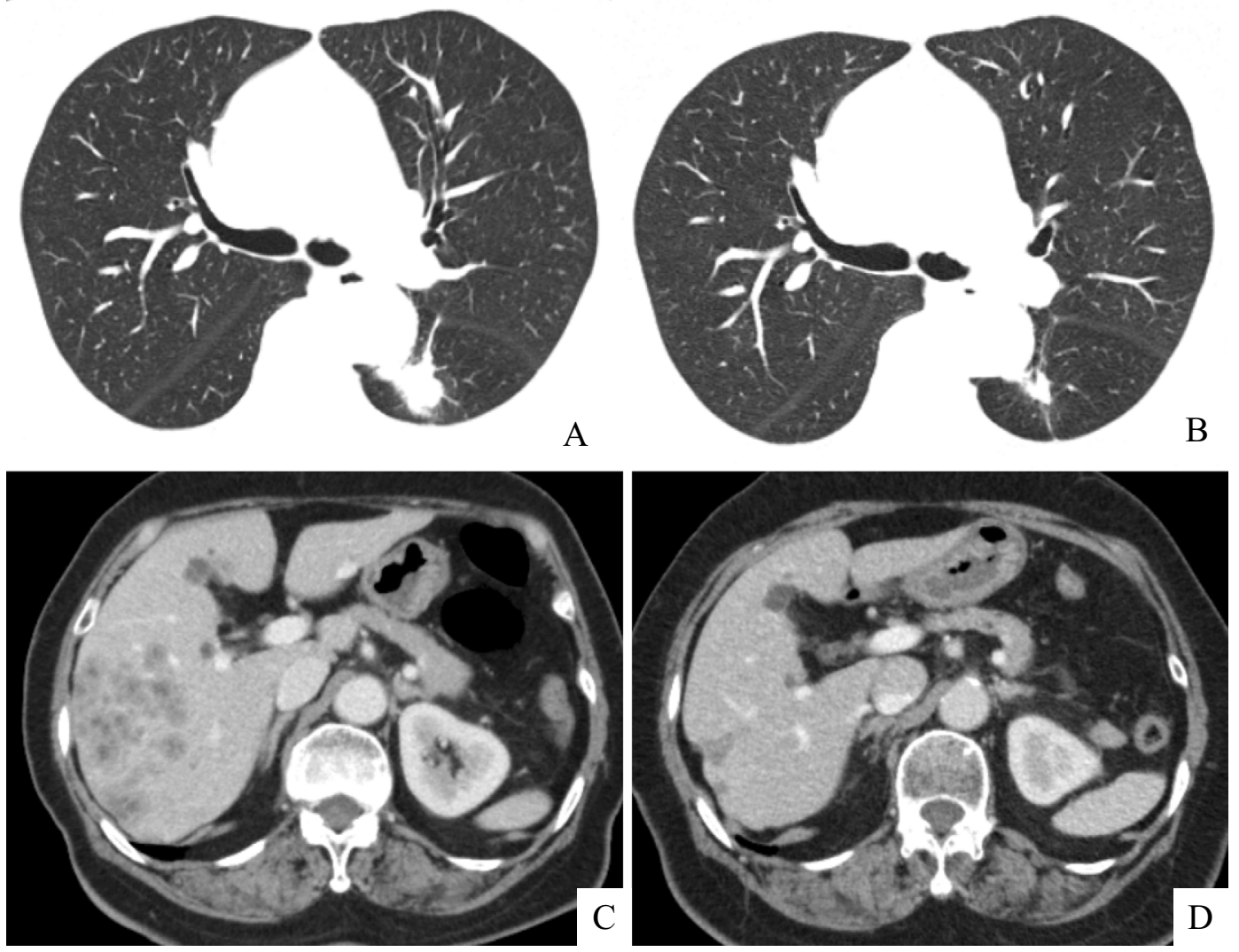

Figure 1. Chest computed tomography revealed a 21-mm-large, lobulated tumor in the left lower lobe (A). Abdominal computed tomography revealed multiple liver metastases (B). The size of lung tumor reduced from $21 \mathrm{~mm}$ to $16 \mathrm{~mm}(\mathrm{C})$, and the liver metastasis exhibited regression (D) after 4 months of treatment with alectinib.
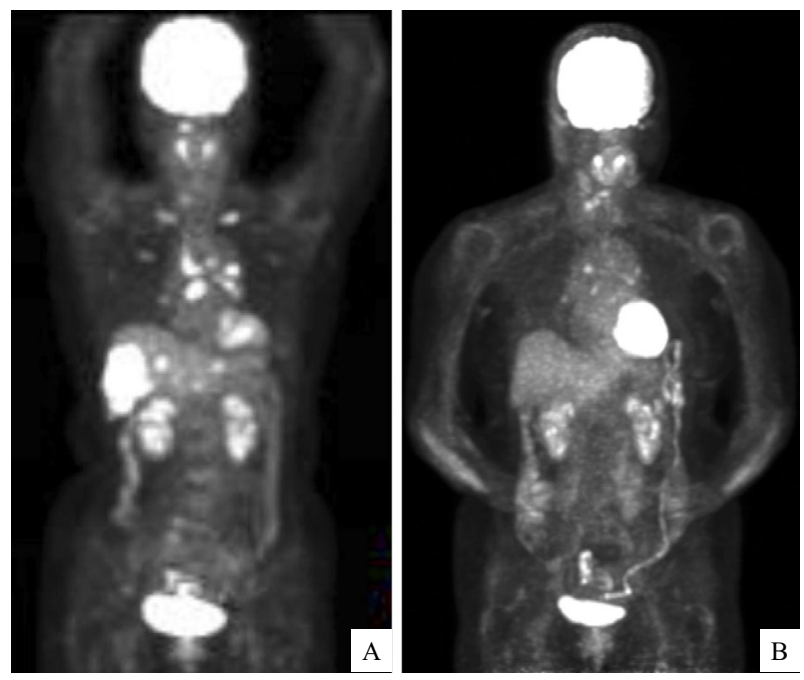

Figure 2. ${ }^{18} \mathrm{~F}$-fluorodeoxyglucose positron emission tomography $\left({ }^{18}\right.$ F-FDG PET) before and after treatment with alectinib. Maximum-intensity projection image from ${ }^{18}$ F-FDG PET shows an intense radiotracer uptake in the left lower lobe, left hilar lymph node, medial lymph node, ribs on both sides, and liver (A). Maximum-intensity projection image from ${ }^{18}$ F-FDG PET shows a weak uptake in only the left hilar lymph node and medial lymph node but disappearance of the uptake in the ribs on both sides and the liver six months after the patient received alectinib (B). the tumor was pathologically diagnosed as LCNEC, and the clinical stage was determined to be stage IV (cT1cN2M1c).

As the performance status of the patient was 2, one cycle of chemotherapy with carboplatin (CBDCA) and irinotecan Camptothecin (CPT-11) was administered as the first-line regimen. The patient's NSE and ProGRP levels were increased (Fig. 4). Although one cycle of chemotherapy with CBDCA and etoposide (VP-16) was administered as the second-line regimen, the NSE and ProGRP levels continued to increase; this increase was considered a characteristic of progressive disease. Four cycles of chemotherapy with amrubicin (AMR) as the third-line regimen reduced the NSE and ProGRP levels; this decrease was considered a characteristic of stable disease. After the fifth cycle of chemotherapy with AMR, the NSE and ProGRP levels slightly increased (Fig. 4). Seven months after the initial diagnosis, a genetic test was performed. Although the epidermal growth factor receptor (EGFR) mutation status of the tumor was wild-type, a fluorescence in situ hybridization analysis with break-apart probes for the ALK gene indicated the presence of an ALK rearrangement in the tumor (Fig. 3F). The patient was treated with alectinib, an ALK tyrosine kinase inhibitor. Four weeks later, the NSE and ProGRP levels were within the normal range. After 4 months of treatment with alectinib, the lung tumor shrank in size from $21 \mathrm{~mm}$ to 16 $\mathrm{mm}$ (Fig. 1C), and the liver metastasis exhibited regression 

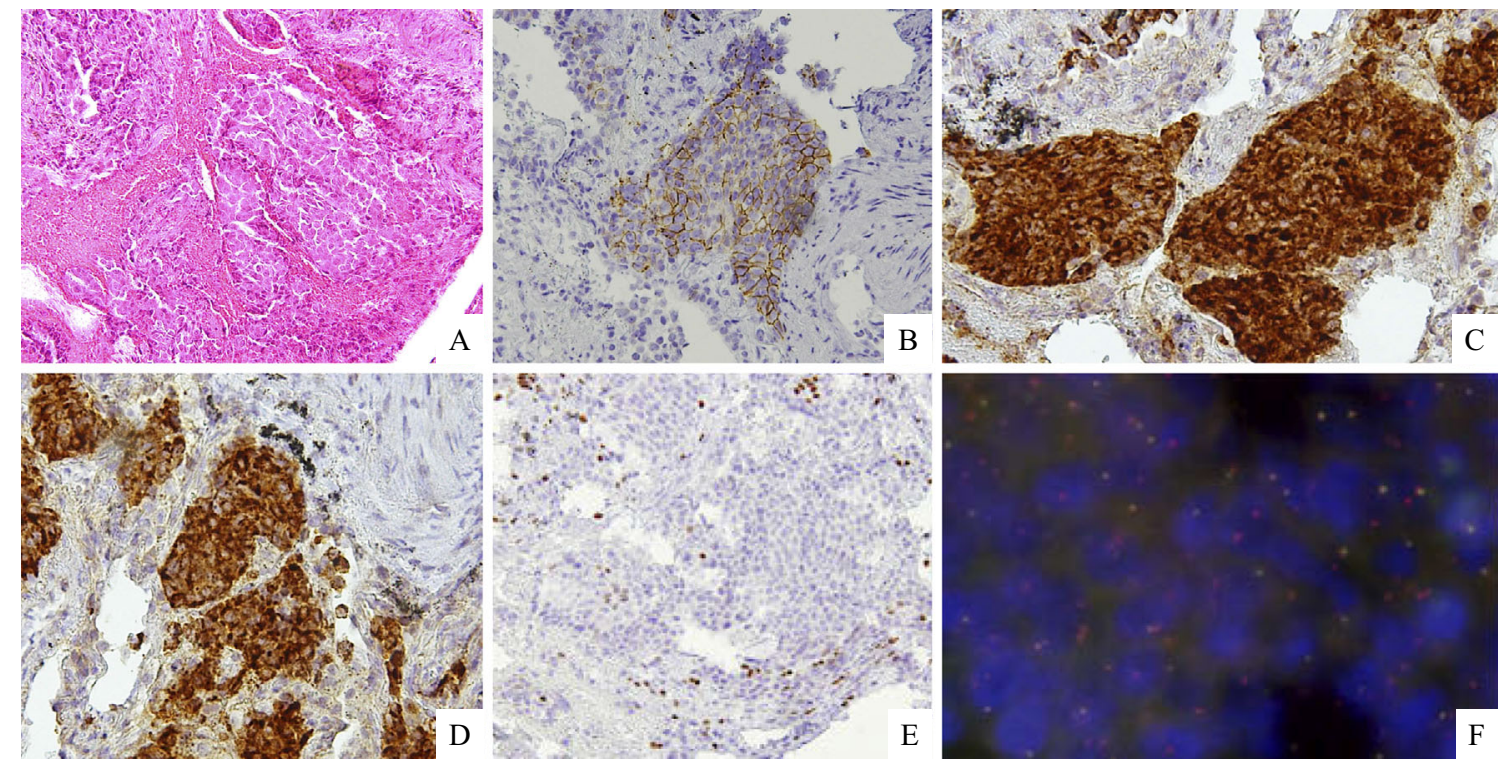

Figure 3. On Hematoxylin and Eosin staining, a transbronchial lung biopsy specimen from the lower lobe revealed malignant neoplasia organized into solid nests of tumor cells with moderately abundant cytoplasm (A). In immunohistochemical studies, tumor cells tested positive for CD56 (B), chromogranin (C), and synaptophysin (D) and negative for thyroid transcription factor-1 (TTF-1) (E). A fluorescence in situ hybridization analysis of the ALK locus indicated the presence of an ALK rearrangement because most tumor cells showed a red signal as the 3'ALK probe.

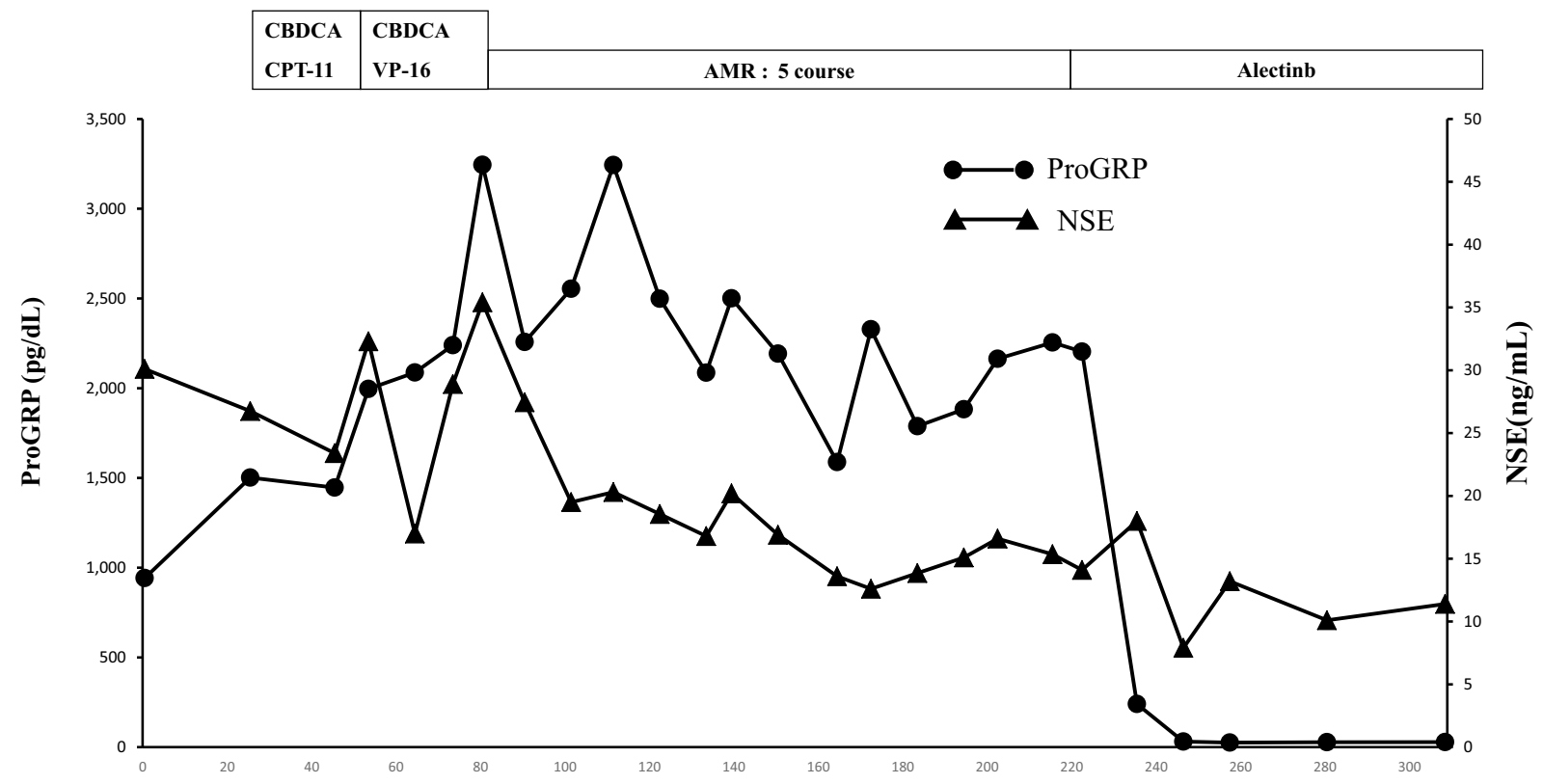

Figure 4. Clinical course

(Fig. 1D). The tumor response was evaluated and considered a partial response.

Treatment-related adverse events included mild constipation and fatigue, but they were tolerable. Six months after treatment with alectinib, the NSE and ProGRP levels remained within normal range (Fig. 4). A maximum-intensity projection image from ${ }^{18} \mathrm{~F}$-FDG PET demonstrated no uptake in the ribs on either side or in the liver, a significant improvement after treatment with alectinib (Fig. 2B).

\section{Discussion}

Gene mutations in tyrosine kinases play essential roles in the pathogenesis of lung adenocarcinoma. Tumors harboring an ALK rearrangement can be successfully treated by ALK inhibitors.

ALK rearrangement is most commonly observed in lung adenocarcinoma, a subset of NSCLC (1). Yoshida et al. reported that ALK-positive cancers commonly express TTF- 
1 (6). This case was pathologically diagnosed as LCNEC with no involvement of lung adenocarcinoma component. The tumors tested positive for immunohistochemical reactions of neuroendocrine markers (CD56, synaptophysin, and chromogranin) and negative for immunohistochemical reactions of TTF-1. Two cases of LCNEC harboring an ALK rearrangement have been reported to date. The first case was that of a 43-year-old never-smoker woman who received crizotinib, an ALK tyrosine kinase inhibitor (4). The second case was that of a 60-year-old never-smoker woman who received no ALK inhibitor (5). Although the lung tumors in the first case remained stable with crizotinib treatment, the brain metastasis enlarged, indicating tumor resistance to crizotinib. Alectinib has been shown to have important clinical activity in brain metastases (7), with an improved progression-free survival compared with that achieved with crizotinib (8). We therefore administered alectinib, and it reduced the tumors in the lung and liver.

Pulmonary neuroendocrine carcinoma includes LCNEC, SCLC, and carcinoid tumor. To date, only two cases of SCLC harboring the echinoderm microtubule-associated protein-like (EML)4-ALK fusion gene have been reported $(9,10)$. These cases were those of a 72-year-old woman and a 43-year-old woman had who received no ALK inhibitors. Three atypical carcinoid tumors harboring the EML4-ALK fusion gene have been reported to date (11-13). The first case was of a 54-year-old woman who exhibited resistance to crizotinib (11). The second case was of a 70year-old man who was successfully treated with cizotinib (12), and the third case was of a 52-year-old man who was successfully treated with alectinib (13). Further studies are required to identify the ALK inhibitors that may be become a key tool for treating pulmonary neuroendocrine carcinoma harboring the EML4-ALK fusion.

The limitation of the present case is that an immunohistological analysis could not be conducted for ALK. After a genetic analysis of the EGFR mutation and ALK rearrangement for the histological diagnosis, unfortunately, no specimen had been preserved for an ALK immunohistological analysis.

We herein reported a rare case of LCNEC harboring an ALK rearrangement. Although LCNEC harboring an ALK rearrangement in a previous case exhibited resistance to crizotinib, the tumor in the present case demonstrated a response to alectinib.
The authors state that they have no Conflict of Interest (COI).

\section{References}

1. Shaw AT, Kim DW, Nakagawa K, et al. Crizotinib versus chemotherapy in advanced ALK-positive lung cancer. N Engl J Med 368: 2385-2394, 2013.

2. Solomon BJ, Mok T, Kim DW, et al. First-line crizotinib versus chemotherapy in advanced ALK-positive lung cancer. $\mathrm{N}$ Engl J Med 371: 2167-2177, 2014.

3. Fasno M, Della Corte CM, Papaccio F, et al. Pulmonary large-cell neuroendocrine carcinoma. J Thorac Oncol 10: 1133-1141, 2015.

4. Omachi N, Shimizu S, Kawaguchi T, et al. A case of large-cell neuroendocrine carcinoma harboring an EML4-ALK rearrangement with resistance to the ALK inhibitor crizotinib. J Thorac Oncol 9: e40-e42, 2014.

5. Kato M, Higuchi K, Shimojo HA, et al. Cytology of largecell neuroendocrine carcinoma harboring an EML4-ALK rearrangement-a case report. Medical Journal of Aizawa Hospital 13: 57-63, 2015 (in Japanese, Abstract in English).

6. Yoshida A, Tsuta K, Watanabe $\mathrm{S}$, et al. Frequent ALK rearrangement and TTF-1/p63 co-expression in lung adenocarcinoma with signet-ring cell component. Lung Cancer 72: 309-315, 2011.

7. Ou SH, Ahn JS, De Petris L, et al. Alectinib in crizontibrefractory ALK-rearranged non-small-cell lung cancer; a phase II global study. J Clin Oncol 34: 661-668, 2016.

8. Hida T, Nokihara H, Kondo M, et al. Alectinib versus crizotinib in patients with ALK-positive non-small cell lung cancer (J-ALEX): an open-label randomized phase 3 trial. Lancet 390: 29-39, 2017.

9. Toyokawa G, Taguchi K, Ohba T, et al. First case of combined small-cell lung cancer with adenocarcinoma harboring EML4ALK fusion and an exon 19 EGFR mutation in each histological component. J Thorac Oncol 7: e39-e41, 2012.

10. Toyokawa G, Takenoyama M, Taguchi $\mathrm{K}$, et al. An extremely rare case of small-cell lung cancer harboring variant 2 of the EML4ALK fusion gene. Lung Cancer 81: 487-490, 2013.

11. Fukuizumi A, Akagi K, Sakai H, et al. A case of atypical carcinoid harboring variant 3a/b EML4+ALK rearrangement. J Thorac Oncol 10: e104-e106, 2015.

12. Nakajima M, Uchiyama N, Shigemasa R, et al. Atypical carcinoid tumor with anaplastic lymphoma kinase (ALK) rearrangement successfully treated by an ALK inhibitor. Intern Med 55: 3151-3153, 2016.

13. Wang VE, Young L, Ali S, et al. A case of metastatic atypical neuroendocrine tumor with ALK translocation and diffuse metastases. Oncologist 22: 1-6, 2017.

The Internal Medicine is an Open Access article distributed under the Creative Commons Attribution-NonCommercial-NoDerivatives 4.0 International License. To view the details of this license, please visit (https://creativecommons.org/licenses/ by-nc-nd/4.0/).

(C) 2018 The Japanese Society of Internal Medicine Intern Med 57: 713-716, 2018 